\title{
The importance of risk factors for the prediction of patients with invasive pulmonary aspergillosis
}

\author{
Selçuk Kaya ${ }^{1 *}$, Eda Gençalioğlu ${ }^{1}$, Mehmet Sönmez ${ }^{2}$, Ifthar Köksal ${ }^{1}$ \\ ${ }^{1}$ Department of Infectious Diseases and Clinical Microbiology, Faculty of Medicine, Karadeniz Technical University, Trabzon, Turkey \\ 2Department of Hematology, Faculty of Medicine, Karadeniz Technical University, Trabzon, Turkey
}

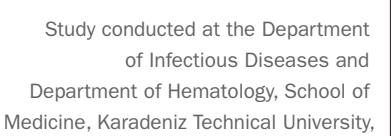

Trabzon, Turkey

Article received: $2 / 5 / 2017$ Accepted for publication: $3 / 1 / 2017$

*Correspondence: Department of Infectious Diseases and Clinical Microbiology, School of Medicine, Karadeniz Technical University, Farabi Hospital Address: Kalkınma street Trabzon - Turkey Postal code: 61080 eselkaya@gmail.com

http://dx.doi.org/10.1590/1806-9282.63.09.764

\section{SUMMARY}

Objective: Invasive pulmonary aspergillosis (IPA) is a major challenge in the management of immunocompromised patients. Despite all the advances in diagnosis, it remains a problem. The purpose of our study was to investigate the risk factors associated with IPA seen in patients with hematological malignancies. Method: A total of 152 febrile neutropenia (FEN) patients with hematological malignancies aged over 18 years and receiving high-dose chemotherapy or stem cell transplant between January 1, 2010, and December 31, 2012 were included in the study. Sixty-five (65) cases with IPA according to the European Organization for the Research and Treatment of Cancer and Infectious Diseases Mycoses Study Group criteria were enrolled as the case group, while 87 patients without IPA development during concomitant monitoring were enrolled as the control group. Incidence of IPA was $21.4 \%(3 / 14)$ in patients receiving bone marrow transplant (allogeneic 2, autologous 1 ) and those cases were also added into the case group. The two groups were compared in terms of demographic, clinical and laboratory findings and risk factors associated with IPA investigated retrospectively.

Results: Presence of relapse of primary disease, neutropenia for more than 3 weeks, presence of bacterial infection, and non-administration of antifungal prophylaxis were identified as risk factors associated with IPA.

Conclusion: It may be possible to reduce the incidence of the disease by eliminating preventable risk factors. Predicting those risks would, per se, enable early diagnosis and treatment and, thus, the mortality rate of these patients would unquestionably decline.

Keywords: invasive pulmonary aspergillosis, hematologic neoplasms, risk factors, early diagnosis, treatment outcome.

\section{INTRODUCTION}

Invasive pulmonary aspergillosis (IPA) is the most common form of invasive aspergillosis and a cause of mortality. The prevalence of IPA has increased in association with the heavy chemotherapy regimens applied in patients with hematological malignancies in recent years. Development of IPA has a negative impact on prognosis as it delays treatment of the patient's primary disease while also causing significant economic losses due to treatment costs for the fungal infection. ${ }^{1,2}$

Various risk factors associated with disease development such as long-term deep neutropenia, long-term broad spectrum antibiotic and corticosteroid use, and allogeneic bone marrow transplant (BMT) have been described. ${ }^{1,2}$ Identification of risk factors is important in terms of preventing the disease developing and allowing early treatment in cases of suspected infection. Thus, based on this idea, we planned to investigate the risk factors for development of IPA in patients with hematological malignancies in the light of the current literature.

\section{Method}

Following clinical research ethical committee approval, the study was performed retrospectively including febrile neutropenic patients with hematological malignancies hospitalized for treatment at the Karadeniz Technical 
University Faculty of Medicine Internal Diseases Department Hematology Clinic.

All patients with febrile neutropenia (FEN) with possible, probable or proven IPA according to European Organization for the Research and Treatment of Cancer and Infectious Diseases Mycoses Study Group (EORTC/MSG) ${ }^{3}$ criteria were enrolled as the case group, while 87 patients without IPA development during concomitant monitoring were enrolled as the control group. The purpose of our study was to investigate risk factors associated with IPA in febrile neutropenic patients with hematological malignancies by comparing the two groups in terms of demographic, clinical and laboratory findings.

\section{Case selection}

\section{Inclusion criteria}

Febrile neutropenic patients aged over 18, with hematological malignancy diagnosed at the Hematology Clinic and who had received high-dose chemotherapy or stem cell transplant were included in the study. MASCC (Multinational Association for Supportive Care in Cancer) scores were lower than 21 in all of the patients.

\section{Exclusion criteria}

Patients undergoing FEN attack during monitoring, who had not undergone high resolution computerized tomography (HRCT) aimed at diagnosis of IPA, and/or without galactomannan (GM) monitoring, and aged under 18 were excluded from the study.

\section{Monitoring of febrile neutropenic patients}

Our hospital's hematology clinic is located on the same floor as the ward for BMT patients. It contained 15 beds, three dormitory-type rooms (each capable of housing four patients), and three transplant rooms with HEPA filters. Only allogeneic BMT patients were followed in transplant rooms. The physical conditions were changed during construction and restoration works between June 2011 and March 2012. During the construction time, the hematology clinic remained in operation at the department opposite the area where the works were taking place, and the number of beds was increased to 16 . However, patients scheduled for BMT were not admitted to the department. Once the construction and repair works were complete, the BMT unit and the hematology department began operating as two distinct units on the same floor. Ward patients transferred to the opposite ward during construction were placed in two-patient rooms. When the construction and repair works had ended, the ward patients continued to be monitored in the same unit. The number of beds in the
BMT unit was increased to seven, and patients began being observed in single rooms with positive pressure. HEPA filters and generally improved physical conditions.

In the light of the EORTC/MSG recommendations, patients' serum GM is studied twice a week, while HRCT is performed on a regular basis. Patients with findings suggestive of IPA are evaluated in terms of bronchoalveolar lavage (BAL) and microbiological and histopathological investigation of specimens obtained from BAL is performed in suitable cases.

\section{Data collection}

Files from 200 patients diagnosed with hematological malignancies at the Internal Diseases department's hematology clinic and subsequently referred to the Infectious Diseases Department following development of FEN in the 3-year period between January 1, 2010 and December 31, 2012 were reviewed retrospectively. Fortyeight (48) patients meeting the exclusion criteria were excluded, and the study proceeded with the remaining 152 patients.

GM values, HRCT reports and microbiological and histopathological report records for patients undergoing BAL were obtained from patient files and the hospital automation system. Demographic and clinical characteristics of the cases were recorded on a "FEN patient with hematological malignancy data form".

\section{Serological tests}

GM antigen was studied over three years using the Sandwich-ELISA method (Platelia ${ }^{\mathrm{TM}}$ Aspergillus, Bio-Rad, France). If the index value was $\geq 0.5$ in consecutive serum specimens or $\geq 0.7$ in a single specimen, and when the GM index in BAL fluid was $\geq 1$, the result was regarded as positive. ${ }^{4,5}$

\section{Radiological tests}

Presence of at least one nodule determined at HRCT, single or multiple nodules and a ground glass appearance due to hemorrhage around these nodules (halo sign), air crescent finding of cavitation suggestive of IPA was regarded as significant for IPA. ${ }^{3,6}$

\section{Invasive tests}

Patients with suspected IPA and regarded as clinically indicated for BAL collection from patient records and the hospital automation system were listed. Microbiological and histopathological assessments of specimens were recorded on the "Febrile neutropenic patient with hematological malignancy data form." 


\section{Patient definition}

Host factors were defined as absolute neutrophil count (ANC) under $500 / \mathrm{mm}^{3}$ and duration of neutropenia exceeding 10 days. $^{3}$

Positivity in serum and BAL values was adopted as a microbiological criterion, while clinical findings such as cough, hemoptysis and chest pain and/or presence of findings in favor of IPA at HRCT were evaluated as clinical criteria. ${ }^{3}$

Based on EORTC/MSG criteria, patients defined as probable or proven IPA were included in the study as the case group.

\section{Statistical analysis}

Risk factors for IPA were identified with single variable analysis, while the Chi-square test $\left(\chi^{2}\right)$ was used for qualitative data. For measurement data, Student's t-test was used for parametric variables and the Mann-Whitney U-test for nonparametric variables. Measurement variables were expressed as mean \pm standard deviation and descriptive data as number and percentage (\%). Analysis results were expressed as $\mathrm{p}$-value, predicted relative risk (odds ratio [OR]) and 95\% confidence interval. Statistical significance was set at $\mathrm{p}<0.05$. Finally logistic regression analysis applied for the variables with significant p-value. Statistical Package for the Social Sciences (SPSS) 13.01 software was used for all analyses.

\section{RESULTS}

Fifty-five (55/36.2\%) of the 152 patients in the study were female and 97 (63.8\%) were male. Mean age of female patients was $46.3 \pm 13.5$ and mean age of male patients 45.0 \pm 15.3 . Patients' demographic characteristics, underlying diseases and the comparison of characteristics and risk factors of patients in the control and case groups are shown in Tables 1 and 2, respectively.

Fourteen (14/9.2\%) of the 152 patients in the study received autologous BMT and 12 (7.8\%) allogeneic BMT. IPA developed+ in only three (21.4\%) patients receiving BMT, two allogeneic and one autologous. BMT did not increase the risk of IPA, the incidence of which was low in patients treated with BMT. Comparison of patients with or without BMT among themselves revealed that acute myeloid leukemia (AML) was the primary diagnosis in $23 \%$ of patients receiving BMT, that the level of accompanying bacterial infection in these patients was $15.3 \%$, and that $15.5 \%$ used multiple antibiotics.

The number of patients receiving antifungal prophylaxis was $19(29.2 \%)$ in the case group and $42(48.2 \%)$ in the control. Fluconazole represented $23 \%$ of the agents used in antifungal prophylaxis and posaconazole represented $77 \%$. The number of patients not receiving antifungal prophylaxis was $46(70.8 \%)$ in the case group and 45 (51.7\%) in the control group. This difference was sta-

\section{TABLE 1 Demographic characteristics and underlying diseases in febrile neutropenic patients.}

\begin{tabular}{|c|c|c|}
\hline Patient characteristics & $n=152$ & Percentage (\%) \\
\hline Female & 55 & 36.2 \\
\hline Mean age $\pm S D(\min -\max )$ & $46.3 \pm 13.5(18-78)$ & \\
\hline Male & 97 & 63.8 \\
\hline Mean age $\pm S D($ min-max $)$ & $45.0 \pm 15.3(18-76)$ & \\
\hline \multicolumn{3}{|l|}{ Underlying hematological malignancy } \\
\hline Acute myeloid leukemia (AML) & 81 & 53.3 \\
\hline Acute lymphoblastic leukemia (ALL) & 26 & 17.1 \\
\hline \multicolumn{3}{|l|}{ Non-Hodgkin lymphoma (NHL) } \\
\hline Multiple myeloma (MM) & 26 & 17.1 \\
\hline Hodgkin lymphoma (HL) & 9 & 5.9 \\
\hline \multicolumn{3}{|l|}{ Count of previous FEN attacks } \\
\hline 0 & 40 & 26.3 \\
\hline 1 & 39 & 25.7 \\
\hline 2 & 24 & 15.8 \\
\hline 3 or more & 49 & 32.2 \\
\hline Number of patients placed in hepa filtered rooms & 12 & 7.8 \\
\hline
\end{tabular}


TABLE 2 Comparison of characteristics and risk factors of patients in the case and control groups.

\begin{tabular}{|c|c|c|c|}
\hline Risk factors & $\begin{array}{l}\text { Case } \\
n=65(\%)\end{array}$ & $\begin{array}{l}\text { Control } \\
n=87(\%)\end{array}$ & $\mathbf{p}$ \\
\hline Age & $44.8 \pm 16.4$ & $60.2 \pm 17.1$ & 0.633 \\
\hline Sex (male/female) & $24 / 41$ & $56 / 31$ & 1.000 \\
\hline \multicolumn{4}{|l|}{ Underlying hematological malignancy } \\
\hline AML & $44(67.7)$ & $37(42.5)$ & 0.002 \\
\hline ALL & $10(15.4)$ & $16(18.4)$ & 0.787 \\
\hline $\mathrm{NHL}$ & $7(10.8)$ & $19(21.8)$ & 0.115 \\
\hline Other & $4(6.2)$ & $15(17.2)$ & 0.072 \\
\hline \multicolumn{4}{|l|}{ Number of previous FEN attacks } \\
\hline 0 & $16(24.6)$ & $24(27.5)$ & 0.821 \\
\hline 1 & $11(16.9)$ & $28(32.1)$ & 0.519 \\
\hline 2 & $10(15.3)$ & $14(16.1)$ & 0.915 \\
\hline 3 or more & $38(58.5)$ & $35(40.2)$ & 0.026 \\
\hline Presence of relapse of primary disease & $36(55.4)$ & $14(16.1)$ & $<0.001$ \\
\hline \multicolumn{4}{|l|}{ Deep neutropenia } \\
\hline MNS $<100 / \mathrm{mm}^{3}$ & $59(90.8)$ & $2(2.3)$ & $<0.001$ \\
\hline Neutropenia of long duration $>3$ weeks & $60(92.3)$ & $43(49.4)$ & $<0.001$ \\
\hline Presence of CMV infection & $18(27.7)$ & $4(4.6)$ & $<0.001$ \\
\hline Presence of bacterial infection & $46(70.8)$ & $9(10.3)$ & $<0.001$ \\
\hline Use of multiple antibiotics (> 3) & $23(35.4)$ & $5(5.8)$ & $<0.001$ \\
\hline Bone marrow transplant & $3(4.6)$ & $23(26.4$ & 0.001 \\
\hline Steroid use & $4(6.2)$ & $22(25.3)$ & 0.004 \\
\hline Non-administration of antifungal prophylaxis & $46(70.8)$ & $45(51.7)$ & 0.027 \\
\hline Hospitalization during the construction works & $18(52.9)$ & $16(47.1)$ & 0.244 \\
\hline
\end{tabular}

FEN: febrile neutropenic; CMV: cytomegalovirus; MNS: ????; AML: acute myeloid leukemia; ALL: acute lymphoblastic leukemia; NHL: non-Hodgkin lymphoma.

tistically significant $(\mathrm{p}=0.027)$ and not receiving antifungal prophylaxis increased the risk of IPA 3.53-fold.

During the construction works, IPA developed in 18 (52.9\%) individuals in the case group and $16(47.1 \%)$ in the control group $(p=0.244)$. The construction works did not influence the incidence of IPA significantly.

Multivariate analysis was carried out for all of the factors identified as positive on univariate analysis. Presence of relapse of primary disease, neutropenia for more than three weeks, and presence of bacterial infections were identified as risk factors associated with IPA. On the other hand, the incidence of IPA was reduced by $80 \%$ with posaconazole prophylaxis (Table 3 ).

\section{Discussion}

Aspergillosis does not vary depending on age, sex or race. ${ }^{1,2,8}$ In agreement with the literature, no statistically significant difference was observed between the two groups in terms of mean age or sex.

Several studies have shown that AML and myelodysplastic syndromes (MDS) are the hematological malignan-
TABLE 3 Risk factors for invasive pulmonary aspergillosis (IPA) (logistic regression analysis).

\begin{tabular}{llll} 
Variables & P & $\begin{array}{l}\text { Odds } \\
\text { ratio }\end{array}$ & $\begin{array}{l}\mathbf{9 5 \%} \text { confidence } \\
\text { interval }\end{array}$ \\
\hline $\begin{array}{l}\text { Presence of relapse of } \\
\text { primary disease }\end{array}$ & 0.036 & 3.22 & $1.07-9.67$ \\
\hline Neutropenia of $>3$ weeks & 0.002 & 7.01 & $2.03-24.15$ \\
\hline Presence of bacterial infection & 0.000 & 11.50 & $3.77-35.03$ \\
\hline Posaconazole prophylaxis & 0.008 & 0.18 & $0.05-0.64$ \\
\hline
\end{tabular}

cies associated with the greatest risk of IPA..$^{8-10}$ AML was the most common primary disease in our study, followed by acute lymphoblastic leukemia (ALL), non-Hodgkin lymphoma (NHL) and other hematological malignancies. No statistically significant difference was determined in terms of risk of IPA among patients with ALL, NHL or other hematological malignancies. In terms of underlying diseases, our results are compatible with the literature.

While there are no previous studies elucidating the relation between number of previous neutropenic attacks and risk of developing IPA, some authors have reported 
that the risk of IPA may rise as the number of FEN attacks increases. ${ }^{11,12}$ No significant difference in terms of risk of IPA was determined in our study comparing patients with zero, one or two previous attacks. However, a statistically significant correlation was determined between a history of three or more previous attacks and IPA. These results were in agreement with those in the literature.

Presence of relapse of primary disease is associated with poor prognosis. More aggressive chemotherapies are applied in this patient group and length of hospitalization is greater. This patient group therefore represents a higher risk of development of IPA. ${ }^{13-15}$ The number of patients with relapse in our case group was significantly higher than in the control group. Presence of relapse of primary disease increased the risk of IPA 3.22-fold. These results were again compatible with the literature.

Sufficient count and function of neutrophils in the circulation are known to be important to keep fungal infections under control. ${ }^{1}$ Deep and prolonged neutropenia is one of the significant risk factors for development of IPA. Deep neutropenia is defined as lower than 100/ $\mathrm{mm}^{3} .^{12,15,16}$ The number of deep neutropenic patients was significantly higher in our case group. This finding is compatible with the literature.

The risk of IPA increases in line with duration of neutropenia. ${ }^{1}$ The frequency at which IPA develops increased by $1 \%$ every day in the first three weeks of neutropenia, rising to $4-5 \%$ after the $5^{\text {th }}$ week..$^{17,18}$ In the literature, presence of neutropenia lasting more than three weeks has been shown to be the most significant risk factor in terms of development of IPA..$^{1,17,18}$ In our study, the number of patients with neutropenia exceeding three weeks was significantly higher in the case group compared to the control group. Neutropenia exceeding three weeks in length increased the risk of IPA 7.01-fold. These findings were compatible with the literature.

Several studies have reported that the risk of IPA increases in patients with cytomegalovius (CMV) infection. ${ }^{16,19}$ The number of patients with CMV in our study was significantly higher in the case group than in the control group. On the other hand, CMV infections may also be due to long-term neutropenia in case group as mentioned in the literature. ${ }^{20}$

Some studies have reported that accompanying bacterial infection in patients with FEN and multiple antibiotic use can lead to the development of IPA by damaging microbial flora. ${ }^{12,16,21}$ In our study, the number of patients with accompanying bacterial infection was higher in the case group than in the control group. Pres- ence of bacterial infection increased the risk of IPA 11.50fold. The number of patients using three or more antibiotics in our study was also significantly higher in the case group. These findings are compatible with the literature. In conclusion, the preparation of a treatment protocol with each center closely observing its own microorganism profile and the patients' antimicrobial treatment being adjusted in the light of that center's epidemiological data, as well as the prevalence and sensitivity profiles of the microorganisms isolated, represents the most rational approach.

Several recent studies have reported that the risk of IPA increases in the presence of chronic obstructive pulmonary disease (COPD). The most important factor predisposing patients with COPD to develop IPA is corticosteroid use. However, the doses and durations of corticosteroid use that constitute a risk are uncertain. ${ }^{15,22}$ Although the number of patients with COPD in this study was higher in the case group than in the control group, the difference was not statistically significant. We attribute this finding to the low number of patients with COPD.

IPA is less common in subjects receiving autologous bone marrow transplant than in those receiving allogeneic bone marrow transplant. The prevalence of IPA in subjects receiving allogeneic bone marrow transplant varies depending on multiple factors such as donor-receiver compatibility. ${ }^{19,23}$ IPA developed in only three of the 26 patients undergoing BMT in this study. Two of these received allogeneic transplant and one autologous. Although this appears to contradict the literature, when we compared our patients with or without BMT in terms of risk factors, the number of patients diagnosed with AML was low in the BMT group, and levels of accompanying bacterial infection and antibiotic use were also low. Moreover, the number of patients who received antifungal prophylaxis was much higher in the BMT group.

Several studies have reported that steroid use leads to the development of IPA. However, the dose and duration of corticosteroid use that would represent a risk are not clear. One meta-analysis investigated the findings of 71 controlled studies and determined that a daily prednisolone dose $<10 \mathrm{mg}$ or cumulative dose $<700 \mathrm{mg}$ did not increase the risk of infectious complication. ${ }^{24}$ Another study reported that a steroid dose $\geq 1 \mathrm{mg} / \mathrm{kg}$ per day over $\geq 21$ days increased the risk of IPA. ${ }^{25}$ Dexamethasone at $40 \mathrm{mg}$ /day for 4-6 days is used as a steroid in some chemotherapy regimens. Statistically significant difference was determined when the two groups were analyzed in terms of the effect of steroid use on risk of IPA. 
The administration of prophylaxis in patients with hematological malignancies is controversial. Different practices can be seen from one hospital to another in the same country. Primary prophylaxis can be applied to patients at high risk (receiving AML, MDS or allogeneic BMT). ${ }^{21}$ The use of posaconazole for IPA prophylaxis in high-risk AML and MDS patients is more effective than fluconazole or itraconazole, and appears as prophylaxis in the IDSA guideline. ${ }^{26,27}$ Publications regarding posaconazole prophylaxis appear promising, and the number of centers applying posaconazole prophylaxis is increasing. ${ }^{21,26-30}$ Antifungal prophylaxis can be given in selected BMT patients similar to solid organ recipients. ${ }^{31,32}$ The application of antifungal prophylaxis in patients undergoing autologous BMT is still controversial, and antifungal prophylaxis is not recommended for this patient group in the IDSA guideline. ${ }^{31}$ Further studies are needed to identify which patient group will in fact benefit from prophylaxis. Since most of our patients were autologous BMT, no antifungal prophylaxis was given to them, which is in accordance with current guidelines. Prophylaxis was administered to $40 \%$ of our patients, with posaconazole in $2 / 3$ of these and fluconazole in the remaining $1 / 3$. The number of patients not receiving antifungal prophylaxis was significantly higher in the case group compared to the control group, and not receiving antifungal prophylaxis increased the risk of IPA 3.53-fold. The incidence of IPA was reduced considerably through the posaconazole prophylaxis.

The presence of Aspergillus spores in hospital environment is an important risk factor for the development of IPA. There is actually a large number of nosocomial outbreaks reported in the literature during construction works. ${ }^{14,33,34}$ There was ongoing construction works for a few months in the current study; however, this did not affect the incidence of IPA importantly.

\section{Conclusion}

It should be kept in mind that reduction of disease incidence may be possible with elimination of preventable risk factors. Due to difficulties in diagnosis, a significant proportion of patients with IPA are diagnosed late, and the disease is often fatal. It is therefore of great importance for patients with multiple risk factors for the development of IPA to be identified and closely monitored, and for diagnostic procedures in these patients to be performed without delay. Success can be improved by teams comprising hematology, infectious diseases and clinical microbiology, radiology and medical microbiology specialists collaborating within a multidisciplinary approach.

\section{References}

1. Chamilos G, Luna M, Lewis RE, Bodey GP, Chemaly R, Tarrand JJ, et al. Invasive fungal infections in patients with hematologic malignancies in a tertiary care center: a autopsy study over a 15-year period (1989-2003). Hematologica. 2006; 91(7):986-9.

2. Patterson TF, Kirkpatrick WR, White M, Hiemenz JW, Wingard JR, Dupont B, et al. Invasive aspergillosis. Disease spectrum, treatment practices, and outcomes. I3 Aspergillus Study Group. Medicine (Baltimore). 2000; 79(4):250-60.

3. De Pauw B, Walsh TJ, Donnelly JP Stevens DA, Edwards JE, Calandra T, et al.; European Organization for Research and Treatment of Cancer/Invasive Fungal Infections Cooperative Group; National Institute of Allergy and Infectious Diseases Mycoses Study Group (EORTC/MSG) Consensus Group. Revised definitions of invasive fungal disease from the European Organization for Research and Treatment of Cancer/Invasive Fungal Infections Cooperative Group and the National Institute of Allergy and Infectious Diseases Mycoses Study Group (EORTC/MSG) Consensus Group. Clin Infect Dis. 2008; 46(12):1813-21.

4. Maertens JA, Klont R, Mason C, Theunissen K, Meersseman W, Lagrou K, et al. Optimization of the cutoff value for the Aspergillus double-sandwich enzyme immunoassay. Clin Infect Dis. 2007; 44(10):1329-36.

5. Koo S, Bryar JM, Baden LR, Marty FM. Prognostic features of galactomannan antigenemia in galactomannan-positive invasive aspergillosis. J Clin Microb. 2010; 48(4):1255-60.

6. Greene R. The radiological spectrum of pulmonary aspergillosis. Med Mycol. 2005; 43(Suppl 1):S147-54.

7. Sharma A, Lokeshwar N. Febrile neutropenia in haematological malignancies. J Postgrad Med. 2005; 51(Suppl 1):S42-8.

8. Cornillet A, Camus C, Nimubona S, Gandemer V, Tattevin P, Belleguic C, et al. Comparison of epidemiological, clinical, and biological features of invasive aspergillosis in neutropenic and non neutropenic patients: a 6-year survey. Clin Infect Dis. 2006; 43(5):577-84.

9. Perkhofer S, Lass-Flörl C, Hell M, Russ G, Krause R, Hönigl M, et al. The Nationwide Austrian Aspergillus Registry: a prospective data collection on epidemiology, therapy and outcome of invasive mould infections in immunocompromised and/or immunosuppressed patients. Int J Antimicrob Agents. 2010; 36(6):531-6.

10. Patterson TF. Aspergillus species. In: Mandell GL, Bennett JE, Dolin R, editors. Mandell, Douglas, and Bennett's principles and practice of infectious diseases. 8. ed. Philadelphia: Elsevier Churchill Livingstone; 2015. p. 2895-2908.e4

11. Richardson MD. Changing pattern and trends in systemic fungal infections. J Antimicrob Chemother. 2005; 56(Suppl 1):i5-i11.

12. Mühlemann K, Wenger C, Zenhäusern R, Täuber MG. Risk factors for invasive aspergillosis in neutropenic patients with hematologic malignancies. Leukemia. 2005; 19(4):545-50

13. Lortholary O, Gangneux JP, Sitbon K, Lebeau B, de Monbrison F, Le Strat $\mathrm{Y}$, et al.; French Mycosis Study Group. Epidemiological trends in invasive aspergillosis in France: the SAIF network (2005-2007). Clin Microbiol Infect. 2011; 17(12):1882-9.

14. Erol S. [Nosocomial aspergillosis: epidemiology and control]. Mikrobiyol Bul. 2010; 44(2):323-38.

15. Kunter E. Aspergillosis and invasive pulmonary aspergillosis. Turkiye Klinikleri J Thor Surg-Special Topics. 2012; 5:119-23.

16. Garnacho-Montero J, Amaya-Villar R, Ortiz-Leyba C, León C, Alvarez-Lerma F, Nolla-Salas J, et al. Isolation of Aspergillus spp from the respiratory tract in critically ill patients: risk factors, clinical presentation and outcome. Crit Care. 2005; 9(3):R191-9.

17. Soubani AO, Chandrasekar PH. The clinical spectrum of pulmonary aspergillosis. Chest. 2002; 121(6):1988-99.

18. Ho PL, Yuen KY. Aspergillosis in bone marrow transplant recipients. Crit Rev Oncol Hematol. 2000; 34(1):55-69.

19. Marr KA, Carter RA, Boeckh M, Martin P, Corey L. Invasive aspergillosis in allogenic stem cell transplant recipients: changes in epidemiology and risk factors. Blood. 2002; 100(13):4358-66

20. Kanvinde S, Bhargava P, Patwardhan S. Cytomegalovirus infection as a cause of cytopenia after chemotherapy for hematological malignancies. Indian Pediatr. 2013; 50(2):197-201.

21. Maertens J. Evaluating prophylaxis of invasive fungal infections in patients with haematologic malignancies. Eur J Hematol. 2007; 78(4):275-82.

22. Guinea J, Torres-Narbona M, Gijón P, Muñoz P, Pozo F, Peláez T, et al. Pulmonary aspergillosis in patients with chronic obstructive pulmonary disease: incidence, risk factors, and outcome. Clin Microbiol Infect. 2010; 16(7):870-7. 
23. Morgan J, Wannemuehler KA, Marr KA, Hadley S, Kontoyiannis DP, Walsh $\mathrm{TJ}$, et al. Incidence of invasive aspergillosis following hematopoietic stem cell and solid organ transplantation: interim results of a prospective multicenter surveillance program. Med Mycol. 2005; 43(Suppl 1):S49-58.

24. Frey FJ, Speck RF. [Glucocorticoids and infection]. Schweiz Med Wochenschr. $1992 ; 122(5): 137-46$.

25. Grow WB, Moreb JS, Roque D, Manion K, Leather H, Reddy V, et al. Late onset of invasive Aspergillus infection in bone marrow transplant patients at a university hospital. Bone Marrow Transplant. 2002; 29(1):15-9.

26. Bulpa P, Dive A, Sibille Y. Invasive pulmonary aspergillosis in patients with chronic obstructive pulmonary disease. Eur Respir J. 2007; 30(4):782-800.

27. Cornely OA, Maertens J, Winston DJ, Perfect J, Ullmann AJ, Walsh TJ, et al. Posaconazole vs. fluconazole or itraconazole prophylaxis in patients with neutropenia. N Engl J Med. 2007; 356(4):348-59.

28. Kaya S, Sönmez M, Köksal I, Gencalioglu E, Y1lmaz M, Yılmaz G. Comparison of fluconazole and posaconazole for fungal prophylaxis in high-risk patients with hematological malignity. J Microbiol Infect Dis. 2014; 4(1):1-6.

29. Ullmann AJ, Lipton JH, Vesole DH, Chandrasekar P, Langston A, Tarantolo $\mathrm{SR}$, et al. Posaconazole or fluconazole for prophylaxis in severe graft-versushost disease. N Engl J Med. 2007; 356(4):335-47.
30. Metan G, Türe Z, Pala Ç, Kaynar L, Yıldırım A, Elmalı F, et al. A single center experience for antifungal prophylaxis in patients with acute myelogenous leukemia. Indian J Hematol Blood Transfus. 2015; 31(3):339-45.

31. Walsh TJ, Anaissie EJ, Denning DW, Herbrecht R, Kontoyiannis DP, Marr $\mathrm{KA}$, et al.; Infectious Diseases Society of America. Treatment of aspergillosis: clinical practice guidelines of the Infectious Diseases Society of America. Clin Infect Dis. 2015; 46(3):327-60.

32. Şenol S, Kutsoylu OE, Kaya O, Avcı M, Şıkgöz Taşbakan M, Avkan Oğuz VA, et al. Antifungal prophylaxis in solid organ transplant recipients. Mediterr J Infect Microb Antimicrob. 2015; 4:7. Available from: http://www.mjima org/uploads/pdf/pdf_57.pdf.

33. Aktaş SÇ, Temizel F, Batırel A, Uçkun S, Geyik D, Şahin S, et al. [Hospital building and invasive pulmonary aspergillosis: evaluation of 8 cases]. Mediterr J Infect Microb Antimicrob. 2016; (Suppl 1):120. (Türkiye Ekmud Kongresi, 11-15 Mayis 2016. PS-214). Avaliable from: http://www.mjima.org/uploads/ pdf/pdf_80.pdf.

34. Warris A, Verweij PE. Clinical implications of environmental sources for Aspergillus. Med Mycol. 2005; 43(Suppl 1):S59-65. 